\section{The Interplay of Colloidal Organization and Oxo-Cluster Chemistry: Polyoxometalate-Silica Hybrids-Materials with a Nanochemical Function**}

\author{
By Sebastian Polarz,* Bernd Smarsly, Christine Göltner, and \\ Markus Antonietti
}

Polyoxometalates (POMs) of transition metals (e.g., molybdenum, vanadium) have been a center of research interest for several decades, as they exhibit a great variety of structures and a rich diversity of fascinating properties. ${ }^{[1]}$ The structures range from relatively simple, like the Keggin ion, ${ }^{[2]}$ to extremely complex, such as the wheel-shaped ${ }^{[3-5]}$ and ballshaped molybdate clusters ${ }^{[6]}$ prepared by Müller et al., of which the latter possess 154,176, 248, or 132 metal centers per cluster. Their architectures are not only well-defined, relatively easy to produce, and beautiful, but these compounds also exhibit the electronic, electrochemical, magnetic, and optical qualities typical of mixed-valence molybdenum oxide compounds, which creates potential for many applications. ${ }^{[7,8]}$

In most cases, pure POMs are quite difficult to handle from a materials science point of view. For example, the problem of too high a solubility in water is encountered, together with low $\mathrm{pH}$ and redox stability. In addition, the cluster cavities of most POMs are filled with water of crystallization, which cannot be removed without destroying the structures. ${ }^{[9,10]}$ There is also the inherent problem of lacking access to all inner sites

[*] S. Polarz, B. Smarsly, Dr. C. Göltner, Prof. M. Antonietti Max-Planck Institute of Colloids and Interfaces Research Campus Golm D-14424 Potsdam (Germany) E-mail: sebastian.polarz@mpikg-golm.mpg.de

[**] This work has been financed by the Max Planck Society. The Raman measurements have been made in co-operation with Priv. Doz. Dr. W. Hunnius (Freie Universität Berlin, Institut für Chemie/Anorganische Chemie und Analytische Chemie). of the clusters, which is necessary for application of these compounds, for example in catalysis.

There are only a few examples of POMs which possess a permeable structure representing a pore system providing accessibility to the inner surface comparable to those of porous silica. ${ }^{[9-16]}$ The reason for this is simply the fact that POMs are crystalline, and a sol-gel process similar to the synthesis of silica ${ }^{[17,18]}$ is not yet known for the fabrication of molybdenum trioxide. The introduction of POMs into a solgel-derived silica matrix ${ }^{[7,19]}$ or their immobilization in preformed porous materials ${ }^{[20,21]}$ was used to solve these problems. In this publication we present the incorporation of supramolecular molybdenum polyoxometalates into sol-gel-derived silica.

Two types of molybdenum polyoxometalates were synthesized according to the preparation method published by Müller et al. (see Fig. 5): the spherical $\left(\mathrm{NH}_{4}\right)_{42}\left[\mathrm{Mo}_{132} \mathrm{O}_{372}\left(\mathrm{CH}_{3^{-}}\right.\right.$ $\left.\mathrm{COO})_{30}\left(\mathrm{H}_{2} \mathrm{O}\right)_{72}\right] \mathbf{1},{ }^{[6]}$ and the wheel-shaped $\left[\left(\mathrm{MoO}_{3}\right)_{176}\right.$ $\left.\left(\mathrm{H}_{2} \mathrm{O}\right)_{80} \mathrm{H}_{32}\right] 2$. $^{[4]}$ The sol-gel process to produce the POM-silica hybrid material produces amorphous, optically clear objects (monoliths) in variable size and form (see also Fig. 6a). Due to the presence of the polyoxometalate these monoliths are deeply colored.

The higher electron density of POMs, compared with that of the silica matrix, enables X-ray scattering experiments to provide information about the structure as well as the POM cluster distribution in the hybrid material. The small-angle X-ray scattering (SAXS) diffraction pattern of the silica hybrid material containing the spherical molybdenum cluster $\mathbf{1}$ is shown in Figure 1.

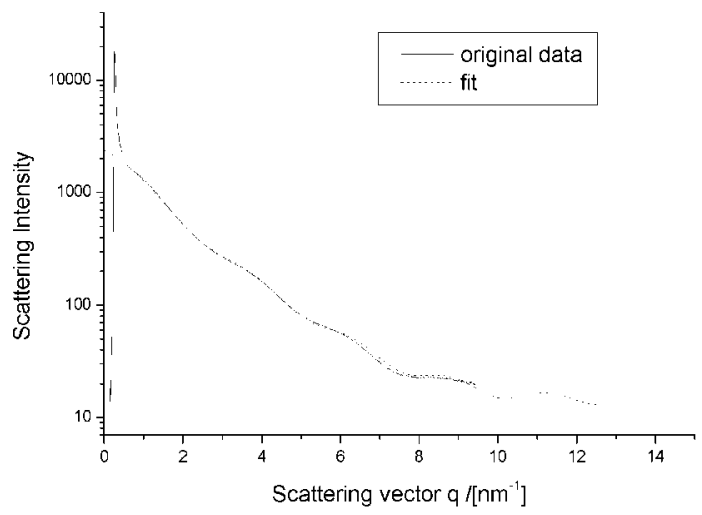

Fig. 1. SAXS diffraction pattern of the 1-silica hybrid material and the fitted curve.

The oscillations and the $q^{-2}$ dependence of the slope are characteristic for monodisperse spherical objects with a hollow shell structure. The increase in the scattering intensity at small $q$ values is due to the primary beam, i.e., there is no indication of further aggregation or any superstructure as was expected since there is no driving force pushing the system into a particularly ordered state. The experimental data were fitted using a model of dilute monodisperse hollow spheres considering the silica matrix as the solvent, see Equation 1. 
$I(q) \propto\left(P(q)+\tilde{I}_{\mathrm{SiO}_{2}}(q)\right)$

Here $P(q)$ is the form factor of a hollow sphere, with the radius and the wall thickness of the hollow sphere as the adjustable parameters, $\tilde{I}_{\mathrm{SiO}_{2}}(q)$ is the background scattering of the $\mathrm{SiO}_{2}$ network (the scattering behavior was estimated from scattering data of an amorphous, non-porous $\mathrm{SiO}_{2}$ sample prepared by a similar sol-gel route) and $q$ is related to the scattering angle by $q=(4 \pi / \lambda) \sin \theta)$.

The fit of the experimental data by the hollow-sphere model with a Gaussian-type electron-density distribution in the wall (thickness $\approx 2 \AA$ ) is very good (Fig. 1) and could not be bettered by other models, such as the massive-sphere model. The clusters are obviously dispersed as single entities in a solution-like fashion.

The experimentally obtained diameter of this hollow sphere $(3.0 \mathrm{~nm})$ fits nicely with the theoretical value for the inner diameter of the ball-shaped POM 1, ${ }^{[6]}$ and the wall-thickness of $2 \AA$ is in good agreement with the molecular structure of $\mathbf{1}$. The diameter is actually slightly too small, but this can be explained by the fact that $\mathbf{1}$ is not "empty" but filled with water which contributes to the electron density.

The shapes of the Raman spectra of $\mathbf{1}$ in the solid state and in the silica hybrid are strikingly similar. The number, sequence, and relative intensities of the bands correlate well with each other. Since the shape of Raman spectra for supramolecular POMs is an indicator for the molecular structure (generally the symmetry is directly related to the vibrational spectrum), it can be concluded that the icosahedral structure of 1 has been preserved during the sol-gel and drying processes. Interestingly, some energies of the vibrational transitions are shifted significantly to lower wavenumbers (see Table 1) as compared with pure $\mathbf{1}^{[6]}$

This phenomenon can be attributed to the fact that the spherical cluster $\mathbf{1}$, trapped within the silica matrix, exists in self-generated pockets or in a kind of "frozen solution". The pocket or cage obviously represents a lower potential well to each individual POM cluster as compared with the force fields existing in the crystal or in aqueous solution.

Consequently the vibrational transitions are shifted to lower energies.

The 1-silica hybrid material shows IR bands (spectra not shown) at $\bar{v}=$ $1635 \mathrm{~cm}^{-1}, 1550 \mathrm{~cm}^{-1}$, and $1420 \mathrm{~cm}^{-1}$, which are higher in comparison with the pure compound $\mathbf{1}\left(\bar{v}=1618 \mathrm{~cm}^{-1}\right.$, $1546 \mathrm{~cm}^{-1}, 1407 \mathrm{~cm}^{-1}$ ). It has to be mentioned that the $\mathrm{SiO}_{2}$ network itself is IR active and, therefore, the absorption bands caused by $\mathbf{1}$ can hardly be distinguished from the noise because of the low molar ratio $\mathbf{1}: \mathrm{SiO}_{2}$. The UV-vis spectrum of the 1-silica hybrid material (adsorption band at $450 \mathrm{~nm}$ ) is identical to the pure 1 spectrum.
Table 1. Comparison of Raman wavenumbers $\left[\mathrm{cm}^{-1}\right]$ of $\mathbf{1}$ and $\mathbf{1}$-silica hybrid material.

\begin{tabular}{cc}
\hline I & $\begin{array}{c}\text { 1-silica } \\
\text { hybrid material }\end{array}$ \\
\hline 951 & 955 \\
876 & 794 \\
564 & 528 \\
468 & 470 \\
374 & 380 \\
321 & 334 \\
211 & 216 \\
\hline
\end{tabular}

Transmission electron microscopy (TEM) can be used to characterize the distribution of the comparatively electrondense POMs within the silica matrix. Figure 2 compares the TEM images of a polycrystalline powder of $\mathbf{1}$ with those of the hybrid material. The TEM picture of the polycrystalline polyoxometalate $\mathbf{1}$ clearly shows a periodic structure with an average spacing of $3.5( \pm 0.5) \mathrm{nm}$. At very thin parts of the specimen, one can even identify single clusters of approximately $3 \mathrm{~nm}$ diameter, which correlates well with the dimensions of the spherical POM 1 as reported in the literature ${ }^{[6]}$ and revealed by the SAXS measurement. To our knowledge, this is the first time this molybdenum cluster has been characterized by an imaging method.

In contrast, the TEM image of the 1-silica hybrid material looks quite homogenous, as expected for the low ratio of POM:SiO $\mathrm{S}_{2}$ However, darker spots that are evenly and statistically distributed within the $\mathrm{SiO}_{2}$ material can be observed. These spots show again diameters of 3-4 nm. By the analytical methods mentioned we have shown that all molecular properties of $\mathbf{1}$ are preserved during the sol-gel condensation (which was furthermore performed in the $\mathrm{pH}$ stability region of $\mathbf{1}$, so that hydrolysis is excluded), including spherical shape, size, monodispersity (SAXS, TEM), vibrations (Raman, IR), and electronic structure (UV-vis). Thus compound $\mathbf{1}$ has not decomposed but is embedded in the silica in its own pores. The latter are homogeneously distributed within the material, which then seems to behave as a solid solution.

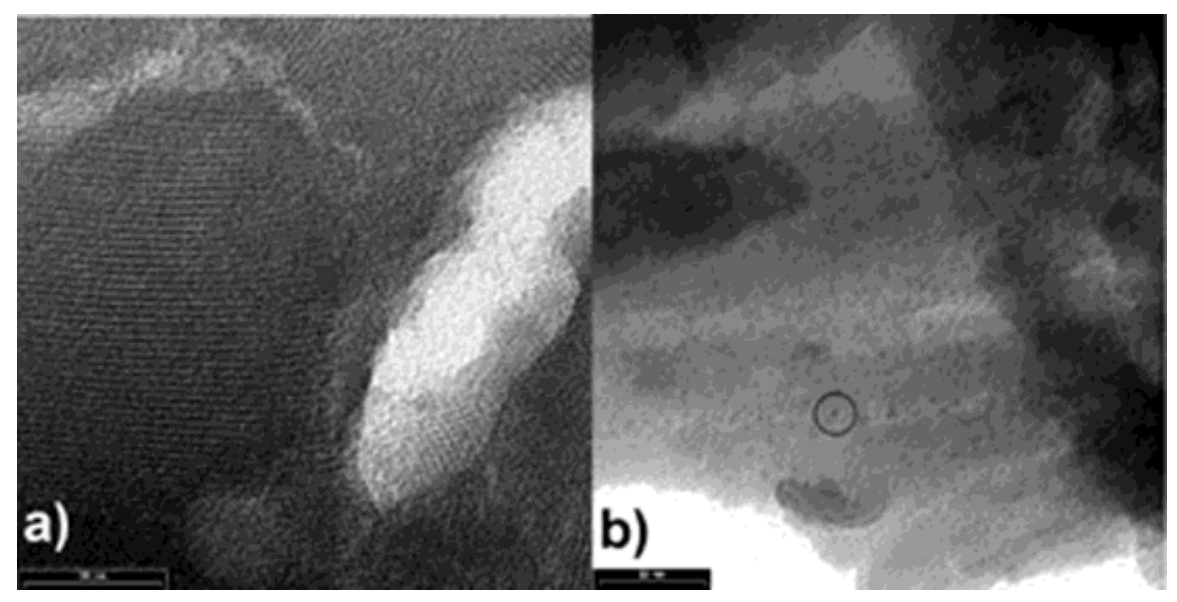

Fig. 2. TEM images of a) pure $\mathbf{1}$ crystals and b) the POM-silica hybrid material. The apparent polydispersity is caused by different planes of observation of the spherical 1 in the silica matrix and is not due to a real polydispersity. Each scalebar represents $50 \mathrm{~nm}$. 
The immobilization of the wheel-shaped POM 2 was investigated by SAXS (Fig. 3) and TEM (Fig. 4). The experimental SAXS data were fitted using a model of monodisperse, dilute rings with the silica matrix as the solvent. In the scattering range accessible by the experimental setup used, the fit correlates well with the experimental data; as only two maxima of scattering intensity are detected in this range, the diameter can only be estimated to be around $4.0 \pm 0.5 \mathrm{~nm}$. It is in addition evident that the slope follows a $q^{-1}$ dependence and is therefore consistent with torus-shaped objects with a Gaussian-type electron-density distribution. The SAXS results are again validated by the TEM images of the $\mathbf{2}$-silica hybrid material (Fig. 4). In addition, statistically distributed darker spots can be recognized in the silica matrix. These spots are not spherical in shape but seem to correspond to disk-like particles with a diameter of $4( \pm 1) \mathrm{nm}$ and a height of $2( \pm 1) \mathrm{nm}$. These values support the scattering data and are in good agreement with the dimensions of pure $\mathbf{2}$ as reported. ${ }^{[4]}$

The experiments presented so far serve to demonstrate that the incorporation of the polyoxometalate clusters is possible whilst preserving their structural identity. However, in the resulting hybrid materials, the clusters exist in a sealed-off state, i.e., they are practically inaccessible to chemical reactants or solvents. The ultimate aim of our work is to produce POMsilica hybrid materials that are sufficiently porous to allow accessibility, but with the pores still being small enough that leaching of the inorganic clusters is prevented. From a synthetic point of view, this can be established by adding an additional porogen creating micropores in the cluster-containing silica.

It has been known from previous experiments that poly(ethylene glycol) (PEG), when introduced into the liquid solgel system, creates micropores whose size depends on the amount of porogen present. ${ }^{[2]}$ The addition of PEG $\left(M_{W}\right.$ $200 \mathrm{~g} / \mathrm{mol}$ ) to the reaction mixture consisting of aqueous POM solution, tetramethylorthosilicate (TMOS), and $\mathrm{HCl}$ does not cause macroscopic phase separation, nor does it affect the structural integrity of the molybdenum clusters.

The TEM images of such a bimodal porous 1-silica hybrid material indicate the existence of micropores (not shown here). The ability of PEG200 to introduce high porosity into the material is further confirmed by nitrogen-sorption measurements. For the pure POM-silica hybrid material, no substantial specific surface area is detected, whereas the addition of 10 or $15 \%$ of PEG200 results in specific surface areas of 300 or $350 \mathrm{~m}^{2} / \mathrm{g}$ respectively, according to five-point measurements. A quantitative evaluation of the SAXS data also proves the existence of an additional transport pore system that shows a mean diameter of about $1 \mathrm{~nm}$. These pores are significantly smaller than those created by the POM cluster and therefore serve excellently in

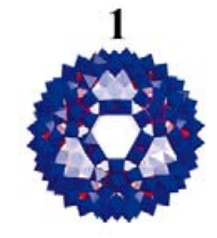

POM

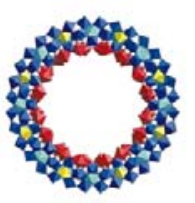

2

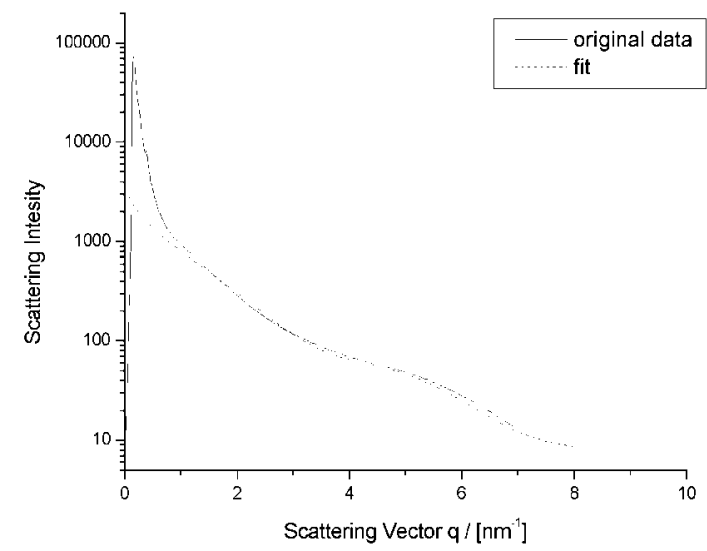

Fig. 3. SAXS diffraction pattern of the $\mathbf{2}$-silica hybrid material and the fitted curve.

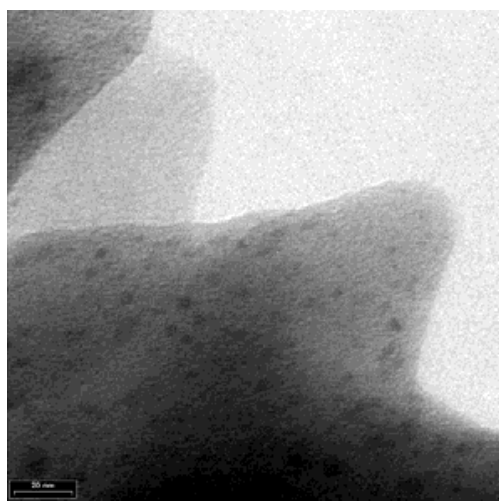

Fig. 4. TEM image of the $\mathbf{2}$-silica hybrid material.

maintaining the molybdenum species and its later fragments or conversion products inside the porous matrix.

For better visualization, the structure of the different types of hybrid systems, as revealed by the different analytical techniques, are sketched in Figure 5. As expected, the POM inside

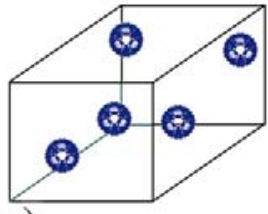

a)

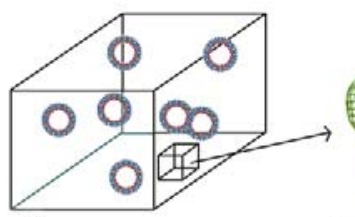

d)

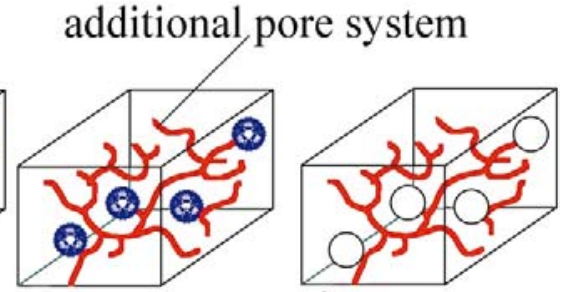

b) c) e)

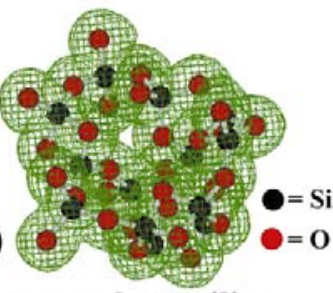

Fig. 5. Schematic presentation of the prepared POM-silica hybrid materials: a) 1-silica hybrid material; b) 1-silica hybrid material with additional micropores; c) resulting material after POM removal; d) 2-silica hybrid material; e) $\mathrm{SiO}_{2}$ random network. 


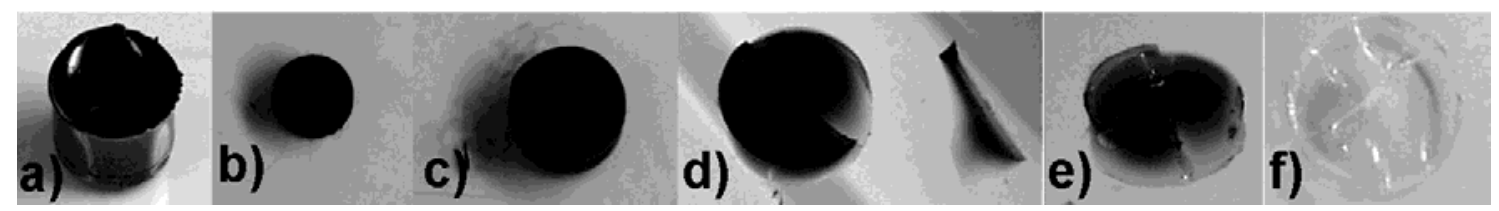

Fig. 6. Reaction of the non-porous 1-silica hybrid material with hydrochloric acid (15\%) a) $t=0 \mathrm{~s} ; \mathrm{b}) t=30 \mathrm{~s} ; \mathrm{c}) t=5 \mathrm{~min} ; \mathrm{d}) t=75 \mathrm{~min}$; e) $t=135 \mathrm{~min}$;) $t=10 \mathrm{~h}$.

the silica matrix can be chemically accessed from the outside medium. With respect to shape, size, and contents, the pores generated by the POM clusters represent a well-defined, monodisperse reaction system that changes neither its shape nor the amount of metal centers in its cavity.

As bulk materials as well as in solution, POMs generally undergo decomposition (in basic $\mathrm{pH}$ media), recondensation (in acidic $\mathrm{pH}$ media), oxidation (e.g., through calcination in air), and reduction (e.g., by reduction with hydrazinium chloride). All these chemical reactions can be performed with the POM in the silica as is clearly shown by the reaction with strong acids which gives rise to a slow change of color from brown via blue and green to colorless, see the series of photographs in Figure 6. According to UV-vis spectra the occurrence of the green hue in the monolith is due to the contemporaneous existence of a blue and a yellow species, which proves the preceding oxidation process (the yellow part is contributed by $\mathrm{MoO}_{3}$, while the blue is caused by a mixed-valence molybdenum oxide species with a delocalized, relatively large electronic system (Robin-Day class II)).

The silica network with additional microporosity provides diffusion pathways and access to the POM-containing pores for substances such as hydrazinium hydrochloride acting as a reducing agent. No leaching of the transition metal from the pore system was detected, as the transport channels within the silica matrix were chosen to be smaller than the POM or its degradation products. As the transition metal species are not allowed to diffuse out of the pores in which they are located, there is the same amount of molybdenum in each pore at all stages of oxidation/reduction or degradation/recondensation. In such a nanochemical environment the stoichiometry of each chemical process within the pores is defined. It is the replicated pocket, which makes chemical reactions structurally reversible. The ability to reconstitute the original structure of the cluster is in this case a specific nanochemical effect, ${ }^{\text {[23-29] }}$ and would be observed neither in solution nor in the bulk material. A chemical and physical analysis of isolated molecules is possible with this novel class of inorganic-inorganic hybrid material.

SAXS was applied to prove that the nanochemical environment of the pocket indeed imposes a "conservation law" for the chemical reaction, i.e., that a maximal size and number of molybdato units is kept. For that, we used $\mathbf{1}$ embedded in the silica matrix and checked for the influence of an acid and the coupled oxidation with regard to structural changes of the oxoclusters.

Figure 7 shows the comparison SAXS curves of the untreated 1-silica hybrid material and the corresponding sam-

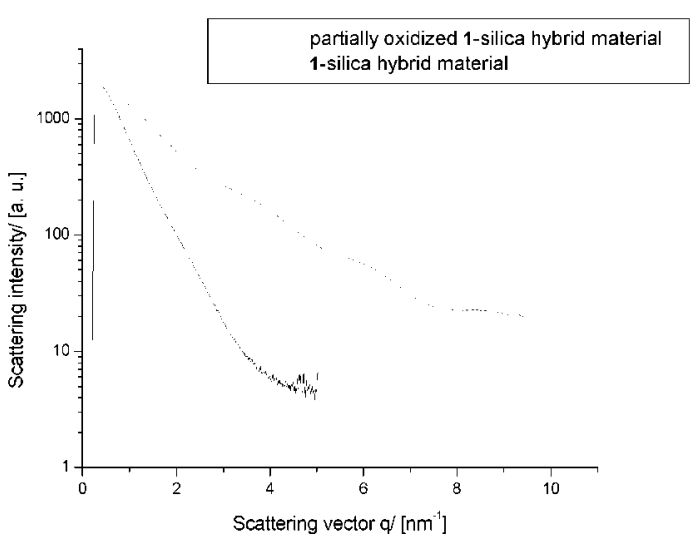

Fig. 7. Diffraction pattern of the 1-silica hybrid material and the corresponding acid-treated and partially oxidized material.

ple after oxidation under otherwise unchanged parameters (recording time, primary intensity, drying conditions, etc.), i.e., a monolith was divided into two parts, one was oxidized and the other kept as a reference. By this procedure a direct comparison of the scattering intensities of the two samples was possible although our experimental SAXS device does not provide data determination in terms of absolute units. It is seen that the absolute scattering intensity extrapolated to vanishing scattering vectors $(q \longrightarrow 0)$ is constant. As in this case the scattering properties are predominantly given by the molybdenum species, the number of heavy metal ions is indeed conserved. Secondly, it is found that both scattering curves switch to a weakly angle-dependent Guinier behavior at about the same scattering vector, i.e., the size of the objects is about the same.

The slopes of the scattering curves describing the shape of reaction product and educt are however significantly different. In contrast to the $q^{-2}$ dependence of the hollow spheres, the intensity decrease of the oxidized sample is much steeper and nearly porod like $\left(\propto q^{-4}\right)$, which is typical for a compact and non-hollow object. It can be assumed that the ball-shaped POMs transform into some less regular, but compact cluster (in excellent agreement with the appearance of a $\mathrm{MoO}_{3}$ species as indicated by the UV-vis measurement) that is restricted to the physical nanospace of the original mold. In other words, there is conservation for the number of metal units and overall size, whereas the shape of the object is allowed to change.

To summarize, it was shown that POMs of colloidal size can be incorporated into silica via a sol-gel process. The system $\mathrm{POM} / \mathrm{SiO}_{2}$ behaves like a solid solution of the POM in $\mathrm{SiO}_{2}$. The structural integrity of the POMs is maintained during and 
after the sol-gel process. The transition-metal oxo clusters are still in possession of all their molecular properties. This opens up new vistas in materials science, because the new hybrid material has the properties of the POM (i.e., unique redox properties) as well as those of the silica (i.e., low solubility in water). In addition, the entrapped POMs are not chemically inert, but are accessible for many reactions in the gas phase (oxidation) or even in solution. Even when distinct microporosity is created within the silica network, the transition metal oxo cluster does not leach from the solid matrix. The materials presented here are the first examples of "nanochemical" conversion of single molecules in an easy-to-handle bulk material.

POM-silica hybrid materials are proposed as potential catalysts, electrochemical sensors (using the 2-silica hybrid material), presumably in the form of thin sol-gel coatings, or tailor-made ceramics ${ }^{[30]}$ with specific magnetic, optical, and electronic properties.

\section{Experimental}

$$
\text { tion. }
$$

All chemicals were obtained from Aldrich and used without further purifica-

Preparation of the POM-Silica Hybrid Materials: A concentrated aqueous solution of the POM $(50 \mathrm{mg} / \mathrm{mL})$ was adjusted to $\mathrm{pH} 4.3$ for $\mathbf{1}$ and $\mathrm{pH} 2.2$ for $\mathbf{2}$ using hydrochloric acid $(1 \mathrm{~N})$. The solution was stirred vigorously, and the required amounts of water and tetramethylorthosilicate (TMOS) were added. In a typical procedure, $0.5 \mathrm{~mL}$ of POM solution were combined with $0.5 \mathrm{~mL}$ of water and $1 \mathrm{~mL}$ of TMOS. When homogenization occurred, stirring was stopped, the reaction vessel sealed with parafilm, and the resulting gels were aged for 5 days. The deeply colored monoliths were dried in air. During drying the monoliths shrank by roughly $40 \%$ in volume, and in some cases cracks appeared.

Preparation of the Porous POM-Silica Hybrid Materials: A concentrated aqueous solution of $\mathbf{1}$ was adjusted to $\mathrm{pH} 4.3$ using hydrochloric acid $(0.1 \mathrm{~N})$. The solution was stirred vigorously, and a controlled amount of PEG was added; TMOS was added after equilibration. In a typical procedure, $50 \mathrm{mg}$ of $\mathbf{1}$ in $1 \mathrm{~mL}$ water, $150 \mathrm{mg}$ PEG200 and $1 \mathrm{~mL}$ TMOS were used. The samples were then treated as described above. The monoliths were finally extracted with acetone for 4 days to remove the PEG.

Characterization of the POM-Silica Hybrid Materials: TEM images were acquired on a Zeiss EM $912 \Omega$ at an acceleration voltage of $120 \mathrm{kV}$. Samples were ground in a ball mill and suspended in acetone. One droplet of the suspension was applied to a 400 mesh carbon-coated copper grid and left to dry in air. SAXS measurements were conducted with a Nonius rotating anode $(P=4 \mathrm{~kW}$, $\mathrm{CuK} \alpha)$ and an image-plate detector system. With the image plates placed at a distance of $40 \mathrm{~cm}$ from the sample, a scattering vector range from $s=0.05$ $1.6 \mathrm{~nm}^{-1}$ was available. The samples were irradiated for $18 \mathrm{~h}$ to reduce the noise level and to obtain a sufficiently high scattering intensity. Two-dimensional diffraction patterns were transformed into a one-dimensional radial average of the scattering intensity. Raman spectra were recorded using a Bruker RFS100 spectrometer. Unground fragments of the monolithic 1-silica hybrid material were used for the measurement. The spectra were obtained with a laser power of $174 \mathrm{~mW}$, a scan number of 3000 and a scan rate of $5 \mathrm{kHz}$. IR spectra were recorded on a Biorad FTS 600 FTIR spectrometer. Unground material fragments were used for the analysis with pure amorphous $\mathrm{SiO}_{2}$ as a background spectrum. Nitrogen sorption isotherms were acquired with a Micromeritics Gemini instrument. The samples for the adsorption measurements were first dried under vacuum at $373 \mathrm{~K}$ for $24 \mathrm{hrs}$. Five-point isotherms were recorded.

Received: April 11, 2000 Final version: June 23, 2000

[1] M. T. Pope, Heteropoly and Isopoly Oxometalates, Springer, Berlin 1983, Vol.1.

[2] J. F. Keggin, Nature 1933, 131, 908

[3] A. Müller, E. Krickemeyer, J. Meyer, H. Bögge, F. Peters, W. Plass, E. Diemann, S. Dillinger, F. Nonnenbruch, M. Randerath, C. Menke, Angew. Chem. 1995, 107, 2293; Angew. Chem. Int. Ed. Engl. 1995, 34, 2122.
[4] A. Müller, E. Krickemeyer, H. Bögge, M. Schmidtmann, C. Beugholt, P. Kögerler, C. Lu, Angew. Chem. 1998, 110, 1278; Angew. Chem. Int. Ed. 1998, 37, 1220.

[5] A. Müller, S. Q. N. Shah, H. Bögge, M. Schmidtmann, Nature 1999, 397, 48.

[6] A. Müller, E. Krickemeyer, H. Bögge, M. Schmidtmann, F. Peters, Angew. Chem. 1998, 110, 3567; Angew. Chem. Int. Ed. 1998, 38, 3359.

[7] D. E. Katsoulis, Chem. Rev. 1998, 98, 359.

[8] A. Müller, M. T. Pope, Polyoxometalates: From Platonic Solids to AntiRetroviral Activity; Topics in Molecular Organization and Engineering, Kluwer, Dordrecht, The Netherlands 1994, Vol.1.

[9] Y. Xu, Curr. Opinion Solid State Mater. Sci. 1999, 4, 133.

[10] T. J. Barton, L. M. Bull, W. G. Klemperer, D. A. Loy, B. McEnaney, M. Misono, P. A. Monson, G. Pez, G. W. Scherer, J. C. Vartuli, O. M. Yaghi, Chem. Mater. 1999, 11, 2633.

[11] J. Y. Ying, C. P. Mehnert, M. S. Wong, Angew. Chem. 1999, 38, 58; Angew. Chem. Int. Ed. 1999, 38, 56.

[12] N. Hüsing, U. Schubert, Angew. Chem. 1998, 110, 22; Angew. Chem. Int. Ed. 1998, 37, 22.

[13] A. Sayari, P. Liu, Microporous Mater. 1997, 12, 149.

[14] D. M. Antonelli, M. Trudeau, Angew. Chem. 1999, 111, 1555; Angew. Chem. Int. Ed. 1999, 38, 1471.

[15] A. K. Cheetham, G. Ferey, T. Loiseau, Angew. Chem. 1999, 111, 3466; Angew. Chem. Int. Ed. 1999, 38, 3268.

[16] W. Dong, B. Dunn, J. Mater. Chem. 1998, 8, 665.

[17] C. J. Brinker, G. W. Scherer, Sol-Gel Science: The Physics and Chemistry of Sol-Gel Processing, Academic, San Diego, CA 1990.

[18] L. L. Hench, J. K. West, Chem. Rev. 1990, 90, 33.

[19] P. Judenstein, H. Schmidt, J. Sol-Gel Sci. Technol. 1994, 3, 189.

[20] C. T. Kresge, D. O. Marler, S. Rav, B. H. Rose, US Patent 5366945,1994

[21] W. Chu, X. Yang, Y. Shan, X. Ye, Y. Wue, Catal. Lett. 1996, 42, 201

[22] B. Smarsly, C. Göltner, B. Berton, M. Antonietti, unpublished.

[23] G. A. Ozin, Adv. Mater. 1992, 4, 612.

[24] K. E. Drexler, Nanosystems; Molecular Machinery, Manufacturing and Computing, Wiley, New York 1992, Vol.1.

[25] J. H. Fendler, Nanoparticles and Nanostructured Films, WILEY-VCH, Weinheim 1998, Vol. 1.

[26] K. Moller, T. Bein, Chem. Mater. 1998, 10, 2950.

[27] L. Dei, M. Mauro, P. Baglioni, Langmuir 1999, 15, 8915.

[28] J. S. Beck, J. C. Vartuli, Curr. Opinion Solid State. Mater. Sci. 1996, 1, 76.

[29] K. Kageyama, J.-I. Tamazawa, T. Aida, Science 1999, 285, 2113.

[30] M. J. MacLachlan, M. Ginzburg, N. Coombs, T. W. Coyle, N. P. Raju, J. E. Greedan, G. A. Ozin, I. Manners, Science 2000, 287, 1460.

\section{Nanoscale Assembly of Metal Clusters in Block Copolymer Films with Vapor of a Metal- Acetylacetonato Complex Using a Dry Process}

\section{By Shin Horiuchi,* Muhammad I. Sarwar, and Yukimichi Nakao}

The organization and patterning of inorganic nanoparticles into two- and three-dimensional (2D and 3D) functional structures at nanometer scale is a potential route to chemical, optical, magnetic, and electronic devices with useful properties. ${ }^{[1]}$ We report a simple and easy method for making nanometer-scale assemblies of metal clusters with a narrow size distribution within the self-assembled microdomain space of block copolymers.

[*] Dr. S. Horiuchi, M. I. Sarwar, Dr. Y. Nakao National Institute of Materials and Chemical Research 1-1, Higashi, Tsukuba, Ibaraki 305-6585 (Japan) E-mail: shoriuchi@nimc.go.jp 